\title{
An Open Source Software for the Measurement of Deformation Fields by Means of Digital Image Correlation
}

\author{
Nicolas Vanderesse ${ }^{1}$, Marin Lagacé ${ }^{2}$, Florent Bridier ${ }^{1}$, Philippe Bocher ${ }^{1}$ \\ 1. École de technologie supérieure, Département de génie mécanique, Montreal, Quebec, Canada \\ 2. Institut de recherche d'Hydro-Québec, Varennes, Quebec, Canada
}

OpenDIC is an application for measuring strain fields observed at the surface of mechanical components. It is based on digital image correlation (DIC), a contactless technique widely used in experimental mechanics. The principle of DIC relies on the comparison of two images of the same specimen before and after applied deformation. It provides the displacement field needed to transform the first image into the second image. The displacements are evaluated over a dense set of independent measurement points. Each point is surrounded by a subset, or a subwindow, that is iteratively compared to subsets in the deformed image until a match is found. By interpolating the deformed image, it is possible to evaluate the displacements with subpixel accuracy. The displacement field is then derived into a deformation field, which is directly related to the actual deformation undergone by the specimen surface [1-3]. Since its creation in the 80 's, DIC has been applied to a variety of problems where contactless strain evaluation is a requirement, with scales ranging from meters to micrometers. Optical images are typically processed, but the last 10 years have seen the increasing use of electronic images $[4,5]$.

Commercial and institutional DIC software products already exist; unfortunately most of them are not easily customizable and upgradable since the details of their implementation are inaccessible and/or poorly documented. OpenDIC, the software presented here, is open source. It has been written on top of RML-Image, an open source image analysis software [6]. It was coded in Java, making it crossplatform. Particular care has been devoted to the documentation for the user, but also for the developer who would need to add functionalities according to specific applications.

OpenDIC yields the displacement field necessary to translate the measurement points from the initial image to corresponding points in the deformed image. The spatial distribution of the points can be adjusted to match specific details in the image (e.g. edges of a specimen). Among other features of the application also figure the automatic treatment of a batch of deformed images with reference to a single initial image, the tuning of parameters such as the subpixel accuracy of the measurements and the interpolation procedure to achieve it, the evaluation of the quality of the results based on the local similarity between the reference and deformed subsets (Fig. 1). A companion application written in MATLAB $^{\circledR}$, DICStrain, can then derive the plane components of the deformation tensor from the displacement field through a finite elements analysis. DICStrain allows for the assessment of deformation heterogeneities by means of tools such as the visualization of strain components, linear profiles and statistics of the strain (Fig. 2).

OpenDIC and DICStrain have been tested with synthetic and real images in order to validate their results and precisely evaluate the causes for possible systematic and random errors. Examples of validation and application of both applications include various mechanical tests on macroscopic metallic specimen and SEM in situ tensile tests revealing microscopic sub-grain strain fields [7]. 
[1] M.A. Sutton et al., Image and Vision Computing, 1 (1983), p. 133.

[2] B. Pan et al., Measurement Science and Technology, 20 (2009), p. 062001.

[3] P. Bornert et al., Experimental Mechanics, 49 (2009), p. 353.

[4] M.A. Sutton et al., Measurement Science and Technology, 17 (2006), p. 2613.

[5] F. Lagattu et al., Materials Characterization, 56 (2006), p. 10.

[6] http://www.rml-image.com, last accessed 2013/02/21.

[7] The authors acknowledge funding from the MITACS Acceleration program.

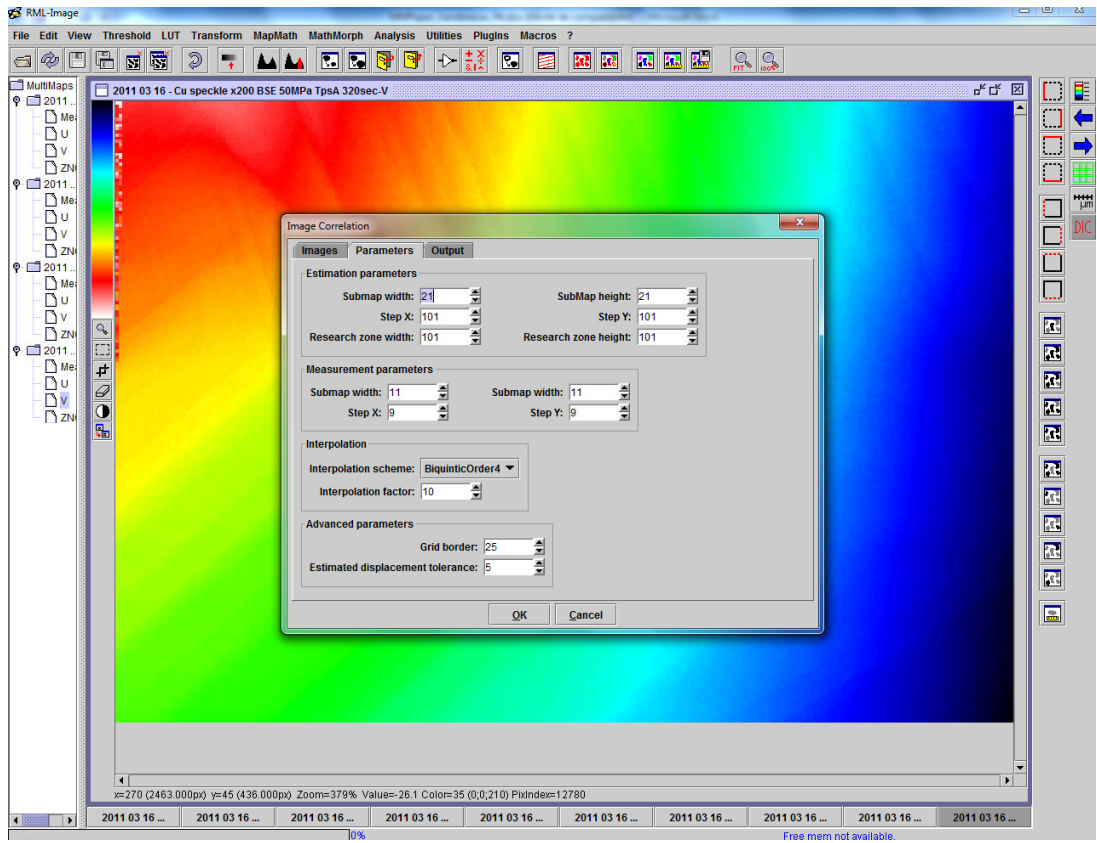

Figure 1. Screenshot of the OpenDIC plugin of RML-Image for measuring displacement fields.

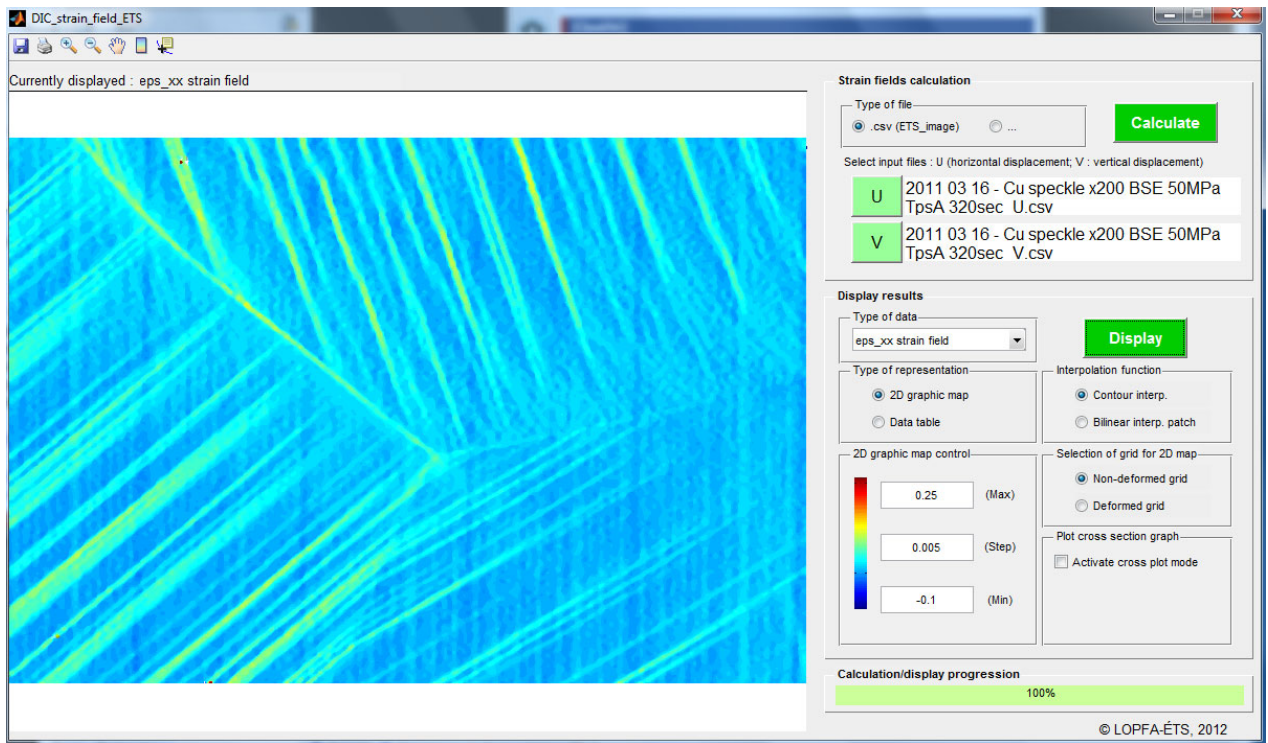

Figure 2. Screenshot of DICStrain for deriving deformation fields. In this example drawn from an in situ experiment with a copper specimen loaded in the SEM, rectilinear strain heterogeneities can be observed. Further investigation shows they are associated to the activity of slip systems. 